\section{Those who are crossing boundaries need less talk, more help and flexibility}

SIR - Interdisciplinary, cross-disciplinary, multidisciplinary and transdisciplinary research are increasingly perceived to be at the frontier of science. But as Adina Payton and Mary Lou Zoback point out in Recruiters ('Crossing boundaries, hitting barriers' Nature 445, 950; 2007), it is not clear how the scientific community can gain from their evolution.

Despite a shift towards an interdisciplinary research culture, we are yet to grapple with how to support a growing number of interdisciplinary researchers. As interdisciplinary postgraduate research students, we face this reality head-on.

We have found it difficult to synthesize the separate perspectives of two or more disciplines into a meaningful middle ground. Unless the scientific community identifies strategies for supporting interdisciplinary researchers to negotiate this middle ground, little progress can be made. Here we suggest two useful approaches.

First, interdisciplinary researchers are expected to develop a different skill set from that of their single-discipline colleagues. In this 'interlocker' role, they engage in a shared conversation between disciplines and work through the tensions this creates. This is more than simply negotiating the different languages and ways of working - it is about appreciating a breadth of knowledge in theory, approach and discourse.

Unfortunately, few systems accommodate this type of researcher - as is sadly demonstrated by emerging frameworks designed to assess research quality in New Zealand, the United Kingdom and Australia. Interdisciplinary committees are needed to assess research proposals, to review grant applications and to examine theses. This would be more effective than the current practice of putting interdisciplinary researchers in assessment 'silos' where they are unrealistically measured against, and by, people in a single discipline.

A second challenge is the disjunct between, on one hand, rhetoric encouraging interdisciplinary research and, on the other, the lack of institutional structure and support for it. Although we are encouraged to work in interdisciplinary environments and to join interdisciplinary research clusters, we face numerous administrative hurdles. Crossenrolment of interdisciplinary students is seldom acknowledged, and adequate resources and structures - such as guidance on writing for interdisciplinary audiences, or longer candidatures for postgraduate students - are rarely provided to support the interdisciplinary researcher.

It would be simple for institutional leaders to ask current interdisciplinary researchers about the challenges they face and to document these issues. These leaders could then address the issues by formalizing the interdisciplinary researcher role and reducing demands to satisfy the needs of multiple disciplines. Supportive environments must be created if we are committed to achieving interdisciplinary research goals.

James A. Smith ${ }^{\star} \uparrow$, Gemma E. Carey ${ }^{\star}+$ *Discipline of Public Health, School of Population Health and Clinical Practice, University of Adelaide tDiscipline of Medicine, School of Medicine, University of Adelaide

Discipline of Anthropology, School of Social Sciences, University of Adelaide, South Australia 5005, Australia

Readers are welcome to comment at http:// blogs.nature.com/nautilus/2007/06/ creating_an_interdisciplinary.html

\section{Limitations of molecular genetics in conservation}

SIR - Your News Feature 'The species and the specious' (Nature 446, 250-253; 2007) provided an interesting assessment of recent research on genetics, species taxonomy and conservation.

Although mitochondrial DNA (mtDNA) and other molecular genetic data are informative, they must be viewed in the context of natural history and population biology. A strictly phylogenetic approach using genetic data may not consider the limitations of gene phylogenies or the relevance of organism-level data. The sciences of systematics, population genetics, phylogenetics and taxonomy require assessment of different types of data. As you note, boundaries between groups within species are not always clear, which has led to extensive assessment of the appropriate units for fish and wildlife management and conservation. I suggest that management should focus on a species' occurrence in geographical areas rather than seemingly endless debate over vague terms such as genetic discreteness or evolutionary legacy, and proliferation of new intraspecific terminology for what are essentially populations.

One example of this debate is provided in your News Feature, in which you note that there is similar mtDNA in polar bears and brown bears that brings their status as species into question. However, morphology, behaviour and habitats show these to be different species regardless of their mtDNA relationship; therefore management of polar bears and brown bears as separate species is appropriate.

The limitations of genetic data are apparent from the contrasting patterns of similar
mtDNA in different species (polar bears and brown bears) and divergent mtDNA within populations of one species, black bears (M. A. Cronin et al. Can. J. Zool. 69, 2985-2992; 1991).

\section{Matthew A. Cronin}

University of Alaska Fairbanks, School of Natural

Resources and Agricultural Sciences,

Palmer Research Center, 533 East Fireweed

Avenue, Palmer, Alaska 99645, USA

\section{Information from patent office could aid replication}

SIR - Your News Feature 'The hard copy' (Nature 446, 485-486; 2007) accurately highlights the limited availability of information on stem-cell research methodologies - owing to competition among labs, the commercial value of such information and space restrictions in high-quality journals - which contributes to other labs' inability to replicate and verify the results.

It might sometimes repay scientists to look beyond conventional journals for information, in this or other disciplines, particularly to patents or patent applications. Thanks to the strict enablement requirements of patent law and patent offices in relation to inventions, one can often find more detailed methodology in patent documents than in journals with severe page limits.

A very good example of comprehensive detail in certain non-embryonic stem-cell methodologies is a PCT application WO/2006/028723 (Non-Embryonic Totipotent Blastomer-Like Stem Cells and Methods Therefor), which includes surgical procedures in organ removal, isolation of cells, and composition and preparation of culture media. In this instance, the level of detail and volume of text relating to methodology far exceeds that which many peer-reviewed journals can accommodate.

Some journals publish methodology and protocols online as Supplementary

Information to the main paper or in separate publications (an example is Nature Protocols, which encourages user comments). Often, though, journals are only starting points in complex paper trails related to methods. In these circumstances, patent documents could contain the most methodology related to an invention in a single document.

\section{Harry Thangaraj}

Centre for the Management of Intellectual Property in Health Research and Development (MIHR), Oxford Centre for Innovation, Mill Street, Oxford OX2 OJX, UK

Science publishing issues of interest to authors are regularly featured at Nautilus (http://blogs.nature.com/nautilus), where we welcome comments and debate. 